Enfoques. Revista de Investigación en Ciencias de la Administración http://doi.org/10.33996/revistaenfoques.v3i9.52 No. 9 | Volumen 3 | Enero - Marzo 2019 http://revistaenfoques.org ISSN: $2016-8219$ pp. $32-48$

\section{ESTRATEGIA DE CONCIENTIZACIÓN PARA LA FORMACIÓN TRIBUTARIA DE LOS ESTUDIANTES DE BACHILLERATO EN VENEZUELA}

\author{
AWARENESS STRATEGY FOR THE TAX EDUCATION OF HIGH \\ SCHOOL STUDENTS IN VENEZUELA
}

Jorge Bordones Rojas, Carlos Caraballo Torrealba y Johany Guevara Patacón

Artículo recibido octubre 2018 | Arbitrado noviembre 2018 | Publicado 01 de enero 2019

\section{Resumen}

El presente artículo destaca la iniciativa de la ejecución de una propuesta acerca de la creación estrategias de formación tributaria para la Unidad Educativa San Miguel Febres Cordero del Municipio Los Guayos, estado Carabobo, Venezuela. La idea surge por la necesidad de que los jóvenes entre 12 y 17 años de edad tengan una formación, educación y cultura tributaria, es decir crear conciencia en materia tributaria. En este sentido, el presente estudio es de campo de tipo descriptivo, dado que, previo estudio y análisis de informaciones contenidas en textos, libros, entre otros, se procederá a efectuar un análisis sistemático de un problema existente en la realidad. La recolección de datos se realizó a través de una encuesta con preguntas cerradas encaminadas a los objetivos propuestos, determinándose la falta de conocimiento en materia tributaria, por lo que se recomienda ejecutar la propuesta estratégica de formación tributaria para los estudiantes de dicha institución realizando juegos, dinámicas y talleres de la materia.

Palabras clave: concientización y formación tributaria; estudiantes de bachillerato

\begin{abstract}
This article highlights the initiative for the execution of a proposal on the creation of tax training strategies for the San Miguel Febres Cordero Educational Unit of the Los Guayos Municipality, Carabobo state, Venezuela. The idea arises from the need for young people between 12 and 17 years of age to have a training, education and tax culture, that is to create awareness in tax matters. In this sense, the present study is a descriptive field, given that, after studying and analyzing information contained in texts, books, among others, a systematic analysis of a problem that exists in reality will proceed. The data collection was carried out through a survey with closed questions aimed at the proposed objectives, determining the lack of knowledge in tax matters, so it is recommended to execute the strategic proposal of tax training for students of said institution performing games, dynamics and workshops of the subject.
\end{abstract}

Key words: tax awareness and training; high school students
Jorge Bordones Rojas

jorgebordones93@hotmail.com Universidad de Carabobo, Venezuela

Licenciado en Contaduría Pública, Universidad De Carabobo, Venezuela. Capacitado en Nominas, Lot $\mathrm{Y}$ Su Reforma. Colegio de Abogados, Contadores y de Relaciones Industriales Herramientas Gerenciales, Grupo Gide Jornadas de Relaciones Industriales, LUTAJS.

Carlos Caraballo Torrealba josecaraballo.1@gmail.com Universidad de Carabobo, Venezuela

Licenciado En Contaduría Pública Universidad de Carabobo. Talleres y cursos realizados Tópicos de Derecho Laboral U.C. Empresas Didácticas INCE.

Johany Guevara Patacón johanyguevara96@gmail.com Universidad de Carabobo, Venezuela Licenciada en Contaduría Pública egresada de la Universidad De Carabobo, Venezuela. 


\section{INTRODUCCIÓN}

Desde hace muchos años, el estado Carabobo ha sido uno de los territorios del país con mayor crecimiento en industrias, siendo beneficiada por su ubicación, sus tierras y por ende su producción, lo cual le ha permitido desarrollarse a nivel económico, pero sin darle importancia a los impuestos, tributos, gravámenes o contribuciones que una persona natural o jurídica puedan realizar al final de cada ejercicio económico, lo cual ha generado desinformación con relación al pago de tributos.

Debido al agotamiento del modelo rentista de desarrollo y del deterioro de las finanzas públicas que dependían en gran medida de ingresos provenientes de las rentas petroleras, el Estado venezolano busca nuevas fuentes de financiamiento, para ello se creó la Superintendencia Nacional de Administración Tributaria (SENIAT), posteriormente reformó un conjunto de leyes entre las que se encuentra la Constitución Nacional (1999), Código Orgánico Tributario (2014), Ley de Impuesto sobre la Renta (2014), Ley de Impuesto al Valor Agregado (2014), entre otras.

Como consecuencia, las personas también han tenido que adecuarse a estas reformas, tomando conciencia que deben cumplir con las obligaciones que contienen las diferentes leyes actualizadas para evitar posibles sanciones y a su vez necesitan conocer sus derechos y garantías para defenderse ante cualquier situación relacionada con el pago de los tributos.

La cultura tributaria nace de la necesidad que tienen los ciudadanos de adquirir información en materia fiscal y la necesidad de que se reciban ingresos no petroleros al Estado.

La cultura tributaria según Méndez (2004),
Se entiende como un conjunto de valores, conocimientos y actitudes compartido por los miembros de una sociedad respecto a la tributación y la observancia de las leyes que la rigen, esto se traduce en una conducta manifestada en el cumplimiento permanente de los deberes tributarios con base en la razón, la confianza y la afirmación de los valores de ética personal, respeto a la ley, responsabilidad ciudadana y solidaridad social de los contribuyentes (p. 123).

Con esta cultura, se pretende que los individuos de la sociedad se involucren en el proceso y puedan tomar conciencia en cuanto al hecho de que es un deber constitucional aportar al Estado y comunicar a la misma colectividad que las razones fundamentales de la tributación son el proporcionar a la Nación los medios necesarios para que éste cumpla con su función primaria, como lo es, la de garantizar a la ciudadanía servicios públicos eficaces y eficientes; así lo establece el artículo 133 de la Constitución de la República Bolivariana de Venezuela: "Toda persona tiene el deber de coadyuvar a los gastos públicos mediante el pago de impuestos, tasa y contribuciones que establezca la ley".

Por su parte, Armas y Colmenares, en la Revista Electrónica de Humanidades, Educación y Comunicación Social de la Universidad Rafael Belloso Chacín en su artículo Educación para el desarrollo de la Cultura Tributaria (2009) destacó que:

La cultura tributaria se identifica con el cumplimiento voluntario de los deberes y obligaciones tributarios por parte del contribuyente y no con la implementación de estrategias para incrementar la recaudación 
de tributos y no bajo presión, por temor a las sanciones establecidas en el Código Orgánico Tributario. (p.144)

De lo antes expuesto, se puede deducir que la existencia de una cultura tributaria, facilita el cumplimiento de los deberes formales, por ello, es de suma importancia el desarrollo y formación desde muy temprana edad en la persona, donde la educación juega un rol importante, para que éste adquiera la creencia firme de cumplir con sus obligaciones tributarias en el futuro, pero el aspecto más trascendente es lograr que esa cultura individual tenga una expresión colectiva.

El desarrollo de programas de educación tributaria, ayudan a la formación de una conciencia y cultura tributaria en toda la población estudiantil, lo cual permitirá alcanzar que las nuevas generaciones cumplan con honestidad, responsabilidad y solidaridad con el pago de los tributos. En este sentido, García Hoz y Medina (1988) dicen, que: "la educación es un proceso personal que depende en primer lugar de las condiciones y actividad del sujeto que se educa, es una tarea viva que está actuando constantemente y se produce en todas las situaciones de la vida".(p. 11).

La educación, al pasar del tiempo, se considera como el recurso más idóneo y el eje principal de todo desarrollo y renovación social. A través del proceso educativo se transmiten los valores esenciales y la preservación de la identidad cultural y ciudadana; siendo la base de la formación y preparación de los recursos humanos necesarios. La escuela es entonces, el lugar para la adquisición y difusión de los conocimientos de relevancia y el medio para el crecimiento de las capacidades productivas.
La Constitución de la República Bolivariana de Venezuela (1999) en su preámbulo, establece un conjunto de principios y políticas con el fin supremo de afianzar una sociedad democrática, participativa y protagónica, en un estado de justicia federal y descentralizada, que asegure entre otros derechos el de la cultura y la educación.

La educación, es el medio a través del cual, se genera el cambio de pensamiento, sentimiento y acción de los ciudadanos, estimulando la participación corresponsable de los individuos para mejorar la calidad de vida del venezolano, de donde surge la necesidad de una relación entre el sector educativo y la administración tributaria, la cual debe desarrollarse en forma armónica, con respeto a los principios constitucionales y una actitud apegada a valores éticos entre los actores participantes.

En este sentido Guaiquirima (2004), afirma lo siguiente: "La formación de la conciencia tributaria no es un trabajo fácil, requiere tiempo, ya que implica un proceso continuo de aprendizaje y asimilación por parte de la sociedad venezolana, siendo necesario el desarrollo, discernimiento y compromiso de todos y cada uno de los miembros del SENIAT, en respaldar la gestión que se realiza. Sobre esa base se deben formular las estrategias de educación tributaria que conduzcan al ciudadano, desde temprana edad, a través de las escuelas, a tomar conciencia de sus responsabilidades para con el Estado". (p. 12).

Para que el Estado pueda cumplir con su obligación constitucional de velar por el bien común y proporcionar a la población los servicios básicos que ésta requiere, necesita de recursos cuya principal fuente son los impuestos pagados por los contribuyentes. El 
pago de los impuestos puede realizarse utilizando el Estado su poder coercitivo contenido en las leyes o apelando a la razón, la cual se manifiesta a través de una cultura tributaria con bases sólidas, lo que evidencia la estrecha relación que existe entre el hecho particular de pagar impuestos, que tiene una trascendencia social, con la cultura tributaria.

Asumiendo que el Estado es garante, por intermedio de la Administración Tributaria, de la divulgación de los deberes y derechos de los contribuyentes, estableciéndolo en el artículo 137 del Código Orgánico Tributario (2014), del cual, se desprende que el mismo, tiene el deber de fomentarla y difundirla a través de sus organismos.

En el estado Carabobo, el nivel de educación secundaria se imparte a los niños y adolescentes, en edades comprendidas entre 12 y 17 años de edad, en este nivel se tiene como propósito organizar la enseñanza y el aprendizaje de contenidos básicos para asegurar que los niños, niñas y adolescentes, adquieran y desarrollen las habilidades intelectuales que les permitan aprender permanentemente y con independencia, así como actuar con eficacia e iniciativa en las cuestiones prácticas de la vida cotidiana, formándose éticamente mediante el conocimiento de sus derechos y sus deberes y la práctica de valores en su vida personal, en sus relaciones con los demás y como integrantes de la comunidad nacional.

Como consecuencia de una incompleta educación tributaria en las Escuelas del Estado Carabobo, el pago de los impuestos se ve inmerso cada día en un mayor incumplimiento voluntario ya que las personas actúan como un patrón ante la sociedad.

Sobre este aspecto, señala Hortta, (1994):
La ética se deriva de la palabra griega ethos que significa "morada", cimentada en la estructura de la persona. Por lo tanto es la ciencia de los actos humanos encaminados hacia el bien, es un conocimiento de la conducta propia dirigida a orientar nuestra actividad hacia el bien, evitando destruirnos. Parte de la persona y se vale del conocimiento de sus actos y termina por elevarla. (p. 36).

El Estado se ve ante el grave problema de lograr el cumplimiento voluntario de la obligación tributaria formal y material, por falta de educación y cultura tributaria, en atención, principalmente, a que los sectores de bajos recursos ven al tributo como una exigencia que se les impone y que agrava aún más su estado de pobreza, puesto que existe en esta sociedad una conciencia de falta de equidad en temas tributarios, existiendo desconfianza generalizada acerca del destino de las sumas recaudadas; se considera que los gobernantes utilizan los fondos para su enriquecimiento personal y no para soportar los gastos públicos, y así brindar una mejor vida a cada uno de los ciudadanos.

La administración tributaria debe estar siempre en la búsqueda de lograr el control en el cumplimiento tributario y el conocimiento y aprobación por parte de los contribuyentes de la normativa legal que regula esta materia, para que posteriormente estos cumplan voluntariamente con sus obligaciones fiscales, dada la importancia que tiene para el país, promover el incremento de la recaudación fiscal lo cual permitirá satisfacer los requerimientos de servicios públicos que demanda la sociedad, cubrir los gastos públicos y disminuir el déficit. 
Es por ello, que debe ser llevado a cabo en todas las escuelas estadales un programa educativo que concientice a la población al pago de los impuestos y a su vez que el Estado a manera de garantizar que los ciudadanos cumplan con su deber tributario ofrezcan detalladamente cuánto, cómo y dónde invirtieron los fondos recaudados para que el contribuyente confíe en el destino de su impuesto.

Para el desarrollo del presente trabajo, se estima que las personas pueden iniciar a moldear una conciencia tributaria y obtener una visión del pago de las obligaciones tributarias a largo plazo desde temprana edad, ya que los valores y los conocimientos más profundos se adquieren durante la primera etapa de vida del ser humano, es por ello que este trabajo tiene por finalidad establecer una propuesta de educación tributaria a las escuelas estadales ubicadas dentro del Municipio los Guayos, considerando que en esta etapa los jóvenes deberían poseer un conocimiento sobre el presente tema, ya que estos serán los futuros contribuyentes del país, con los cuales el Estado podrá contar con una población que ejercite una mayor cultura tributaria.

La presente investigación se llevará a cabo en la Unidad Educativa San Miguel Febres Cordero, que se encuentra ubicada en el Municipio los Guayos del estado Carabobo, con el fin de ayudar a la formación de ciudadanos en proceso de crecimiento desarrollando cultura desde los primeros años de vida involucrándose en el proceso tributario que rige a la sociedad, cumpliendo voluntariamente con las obligaciones tributarias, estableciendo ejemplos para cada venezolano, transmitiendo posteriormente el conocimiento hacia la generación del nuevo ser como hombres y mujeres de nueva extirpe de responsabilidad social, esto a su vez los ayudaría a conocer la importancia del pago de tributos directos e indirectos, ya que poseen establecimientos comerciales cercanos e incluso frente al campo de estudio se encuentra un supermercado Bio donde tienen el deber de pagar el Impuesto al Valor Agregado (IVA) en los productos que le corresponden $y$ en otros casos se presentan las exenciones y exoneraciones del mismo tributo en algunos bienes $y$ servicios.

El aspecto más relevante de esta investigación, es incentivar y conservar la formación de la educación tributaria para alcanzar así una sociedad llena de beneficios a la comunidad. Por ello, se formuló la siguiente interrogante ¿Qué efectos tendría una propuesta de incentivo para la formación tributaria en la población estudiantil de la Unidad Educativa San Miguel Febres Cordero, ubicada dentro del Municipio los Guayos, estado Carabobo?

De acuerdo con los alcances del estudio este tuvo como finalidad proponer estrategias de formación tributaria para la Unidad Educativa San Miguel Febres Cordero ubicada en el Municipio los Guayos, estado Carabobo. Para se diagnosticó el nivel de conocimiento que poseen los niños $y$ adolescentes en materia tributaria, además de analizar los aspectos que impulsan la cultura tributaria en la Unidad Educativa San Miguel Febres Cordero; y por ultimo diseñar las herramientas de formación tributaria para los estudiantes de la Unidad Educativa San Miguel Febres Cordero.

La necesidad de guiar la conducta del contribuyente, para lograr que este adopte la decisión de cumplir voluntariamente con sus obligaciones tributarias conlleva a que se debe estimular el desarrollo de una educación tributaria en la sociedad a través de los medios de difusión, ya que la 
conducta humana se puede modificar y educar transformando los aspectos indeseables en productivos y aceptables por el conjunto social.

A raíz de esta situación, nace la importancia del presente trabajo de investigación que consiste en presentar una propuesta de educación tributaria para las escuelas estadales ubicadas en el Municipio los Guayos, como es el caso de la Unidad Educativa San Miguel Febres Cordero, siendo esta una medida a largo plazo necesaria para disminuir o evitar el incumplimiento de las obligaciones tributarias, ya que se presume que el mismo resulta en parte como consecuencia de la inexistencia de una educación tributaria en la población.

\section{MÉTODO}

El presente estudio se enmarca en la modalidad de investigación cuantitativa, bajo la modalidad de proyecto factible, ya que se analizaron datos numéricos sobre determinadas variables en materia de formación tributaria. Es importante destacar que la investigación cuantitativa se basa en el positivismo, considerándose como un método que se aplica a la investigación científica y filosófica y que presupone la aplicación de instrumentos para la recolección de datos que luego se codificarán, tabularán y se van a analizar para concretar conclusiones. Además, se analizaron documentos relacionados con formación tributaria, estrategias para la formación en educación y diseños herramientas.

\section{RESULTADOS Y DISCUSIÓN}

Una vez aplicado el instrumento de recolección de información, se presenta el análisis general de los resultados:

- Los jóvenes encuestados no tienen conocimientos si existen mecanismos de difusión, tales como volantes, prensa, campañas publicitarias, este resultado constituye una debilidad para gestionar planes y programas vinculados a la participación ciudadana.

- Se puede señalar, que los estudiantes no tienen conocimientos de la importancia del pago de los tributos, puesto que en su mayoría, no tienen una cultura tributaria establecida, pero no porque tienen conciencia de los beneficios que puede traer la recaudación del impuesto a la mejora de los servicios a la comunidad.

- La gran mayoría de los estudiantes desconocen cuáles son los deberes formales que deben cumplir ante la administración tributaria. En este sentido, el nivel de cultura de los contribuyentes incide de manera negativa en la recaudación del impuesto.

- Al respecto, la fomentación de una cultura tributaria que logre cambios en el comportamiento de los contribuyentes es un proceso que necesita tiempo y esfuerzos por parte de las autoridades competentes, ya que la adquisición de un determinado comportamiento cultural en aspectos económicos, sociales y políticos es lento, por lo tanto, si la cultura no se cultiva durante años, no se puede improvisar ni suponer.

- Es necesario, que se realicen campañas para concienciar a los jóvenes de educación secundaria en función de la relevancia de los impuestos, desarrollando una conciencia cívica respecto al cumplimiento tributario, que le permita como contribuyente a futro concebir las obligaciones tributarias 
como un deber y no como una imposición.

\section{Justificación de la propuesta}

La propuesta de herramientas de Formación Tributaria para los estudiantes de la Unidad Educativa San Miguel Febres Cordero, se justifica porque se desarrollarán valores ciudadanos en los niños y jóvenes en edad escolar, poniendo particular énfasis en el vínculo entre tales valores y el sistema tributario, en las consecuencias positivas del cumplimiento tributario $\mathrm{y}$ en las negativas del incumplimiento de los deberes formales. De tal manera, que los alumnos interioricen la idea de la fiscalidad como uno de los ámbitos donde se articula la necesaria correspondencia legal y ética entre derechos y obligaciones, donde se imbrican los intereses personales y los beneficios comunes.

Al mismo tiempo, a través de la formación tributaria se les otorgarán las herramientas propuestas necesarias para formarse una opinión crítica y responsable, con una participación real en el Municipio del que forman parte. Es imprescindible generar en los futuros contribuyentes actitudes de respeto por lo que es público y, en consecuencia, financiado con el esfuerzo de todos y utilizado en beneficio común.
A su vez contribuirá el aumento de la recaudación tributaria, a través de campañas de concientización referidas al conocimiento e importancia que tiene para el Municipio la recaudación de los tributos. Por lo tanto, se hace necesario la implementación de nuevos mecanismos que permitan optimizar la recaudación del impuesto en cuestión para alcanzar las metas de recaudación estimadas, además de brindar un mejor servicio que satisfaga las necesidades de los contribuyentes.

Objetivo 1. Elaborar estrategias para la formación de valores ciudadanos a través de la educación tributaria en la Unidad Educativa San Miguel Febres Cordero

Estas estrategia están orientadas a desarrollar proyectos participativos que deben ser trabajados de una metodología que incentive las buenas prácticas ciudadanas al interior de las instituciones educativas, propiciando un clima democrático en la escuela como condición para el desarrollo de la conciencia ciudadana y tributaria de la Unidad Educativa San Miguel Febres Cordero. Se presenta el siguiente esquema: 


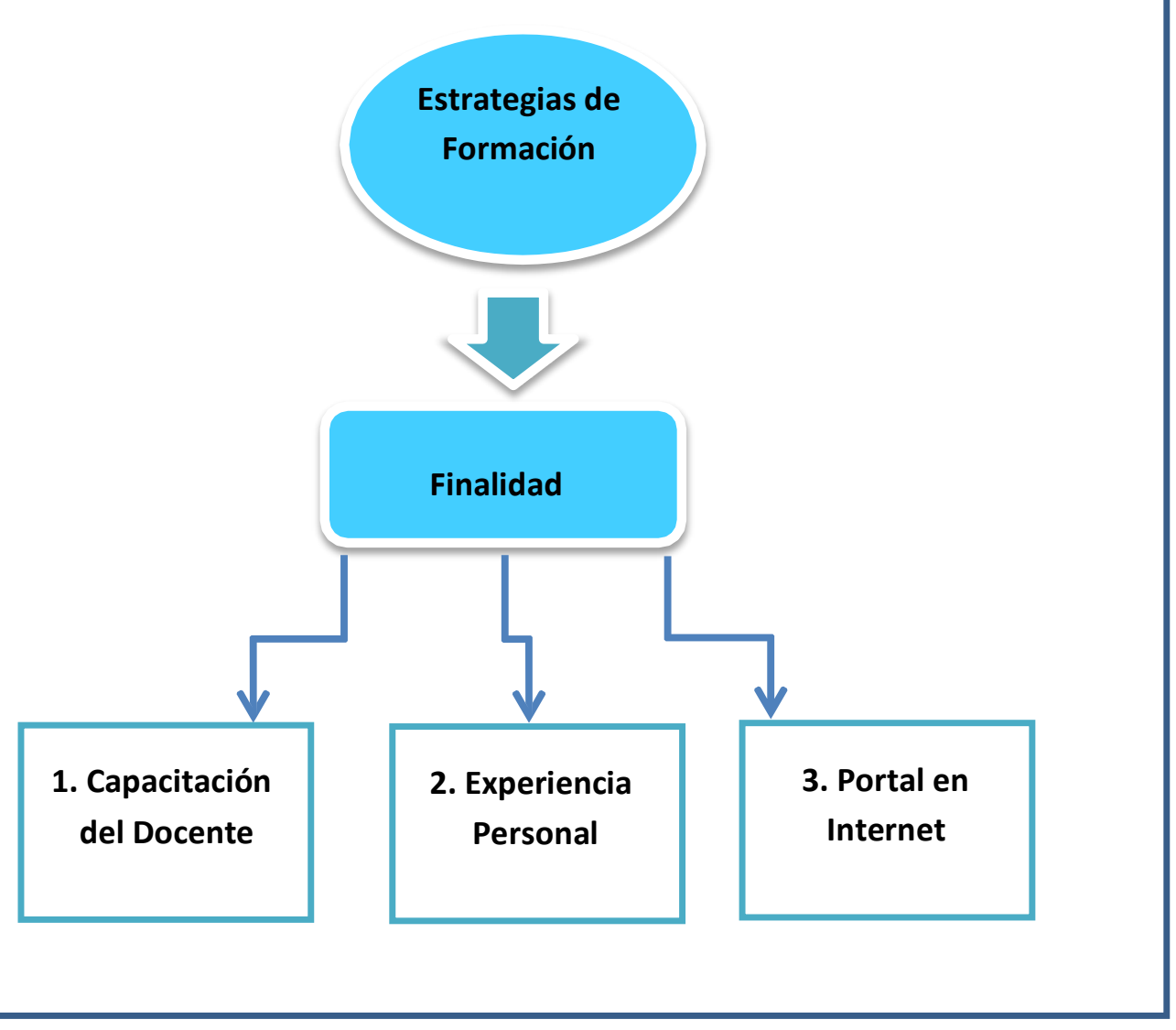

Figura 1. Estrategias de Formación Tributaria. (Fuente: Bordones J, Caraballo C, Guevara J, 2018)

\section{Finalidad}

- Capacitar a los docentes en temas de cultura tributaria, como recurso educativo para la incorporación en los procesos de enseñanza y aprendizaje de los alumnos.

- Fomentar en los estudiantes aprendizajes de cultura tributaria, además de conceptos como: ciudadanía, bienes y servicios públicos, estado, presupuesto, etc. Mediante la práctica de los valores cívicos.

\section{Estrategia 1: capacitación docente}

- Primeramente, se precisa establecer un convenio de cooperación con las autoridades educativas del Ministerio del Poder Popular para la Educación a través de sus Gerencias Regionales de Educación.

- La capacitación de los docentes en formación tributaria serán dictados por la Administración Tributaria.

- Seguidamente, se convocan para el registro e inscripción a los docentes, coordinadores o supervisores que participarán en el programa formación tributaria.

- Los docentes de la Unidad Educativa San Miguel Febres Cordero serán los encargados de ejecutar el programa en la formación tributaria a los estudiantes de la institución educativa. Por lo tanto, se requiere evaluar a los docentes que han participado en la capacitación, otorgándoles una acreditación formal de capacitador. 
- Puesta en práctica de las actividades en las aulas por parte de los docentes capacitados. En esta etapa de ejecución esta labor la ejecutarán los especialistas en las Áreas de Personal Social y Formación Cívica y Ciudadana, quienes formarán una red de especialistas a nivel regional, cuyo objetivo será hacer un seguimiento a los docentes para garantizar que efectivamente ejecuten las actividades en el aula.

\section{Estrategia 2: Experiencia personal}

Se deben realizar visitas guiadas al órgano recaudador (SENIAT). Se trata de acercar a los jóvenes y a sus profesores a la realidad fiscal, mediante la observación directa del funcionamiento de las oficinas de la Agencia Tributaria.

\section{Estrategia 3. Portal en internet}

A través de un portal en Internet, se pone a disposición de los jóvenes y de los profesores una serie de materiales y de recursos que pueden ser utilizados por ellos en cualquier momento, tanto fuera como dentro del aula, para conocer el sentido, el alcance y la finalidad de los impuestos. Ambas actuaciones integran el Programa de Educación Cívico-Tributaria, pero presentan grados de implicación diferentes en cuanto a la planificación, gestión, cobertura, seguimiento y evaluación de cada una de las tareas que las componen.

\section{Objetivo 2: Impulsar mecanismos de divulgación y promoción para los procesos tributarios}

Se propone el uso de herramientas como charlas, seminarios, talleres, acciones de divulgación comunitaria. Es importante que cada herramienta utilizada implique el desarrollo de críticas reflexivas y educacionales, a fin de que el objetivo no se desvirtúa.

Detectar los mecanismos de divulgación adecuados para suministrar la información concerniente a los procesos de fiscalización de los impuestos en el municipio

\section{Acciones}

- Realizar actividades de difusión y publicidad centrada en la importancia de la formación de los ciudadanos, elaborando un registro de información de contribuyentes que permita la ampliación del Padrón de los mismos, pues esto fortalecerá a los ingresos propios del Municipio permitiendo mayor inversión en los servicios a la ciudadanía. Esta estrategia implica la utilización de instrumentos diversos y sencillos de publicidad y difusión.

- Utilizar mecanismos de divulgación, tales como foros sobre las actualizaciones tributarias y tópicos apropiados sobre los procesos de fiscalización, publicidad televisiva y publicidad móvil, apropiados para hacer llegar la información tributaria

\section{Establecer las acciones concretas} realizadas por el docente para impartir un nuevo conocimiento y enlazarlo en la estructura cognoscitiva del alumno.

\section{Acciones:}

- Preparar y alertar al estudiante en relación a qué y cómo va a aprender (activación de conocimientos y experiencias previas pertinentes) permitiéndole ubicarse en el contexto del aprendizaje pertinente.

- Apoyar los contenidos curriculares durante el proceso mismo de aprendizaje. 
Cubren funciones como las siguientes: detección de la información principal; conceptualización de contenidos; delimitación de la organización, entre otros.

\section{Medios de Integración de las Estrategias de Aprendizaje: Comprende el conjunto de medios o vehículos posibles a utilizar para fomentar la educación y el aprendizaje de un conjunto de aspectos relacionados con una temática en específico}

\section{Acciones}

- Establecer acciones a través de medios institucionales realizadas por los entes del gobierno o de organizaciones sin fines de lucro, enfocadas en promover un aprendizaje a través de mensajes que incentivan la participación social.

- Incluir acciones ejecutadas por entes privados, los cuales pueden apoyar con su actividad una labor educativa específica, aportando ideas, recursos económicos e inclusive canales de acceso a la población objetivo.

Objetivo 3: Establecer un Programa de formación, orientación y cultura tributaria para los estudiantes de la Unidad Educativa San Miguel Febres Cordero

\section{Finalidad}

El Estado democrático exige de sus miembros el conocimiento de las normas vigentes como base imprescindible para una convivencia más justa. El sistema tributario es parte integrante de este conjunto de normas. La tributación es el principal recurso económico para el sostenimiento del Estado, por ello es necesario comprender la correspondencia entre los derechos y las obligaciones cívicas y entre intereses privados y beneficios colectivos. Por lo tanto, el programa plantea los siguientes objetivos:

- Desarrollar una conciencia cívica que permita hacer coherentes los comportamientos frente al tributo.

- Adquirir competencias para analizar críticamente la realidad tributaria.

- Reconocer e identificar una norma tributaria y los principios constitucionales sobre tributo.

- Trabajar sobre valores y convivencia, en coincidencia con el diseño curricular de formación ética y ciudadana, aspirando a provocar un impacto cultural que promueva un cambio en el desinterés y apatía existentes por lo público.

- Identificar los bienes y servicios públicos y comprender su valor social y económico.

Población involucrada: los alumnos de cuarto y quinto año de la Unidad Educativa San Miguel Febres Cordero.

Modalidad del Programa: Taller interactivo teórico-práctico Presentación teórica del tema mediante discusiones dirigidas, motivando la participación de los asistentes, aclarando dudas y resolviendo preguntas.

Lugar: Unidad Educativa San Miguel Febres Cordero, se coordinará con las Autoridades de cada colegio lugar, día y hora para el dictado de la clase taller, con el objeto de concentrar a todas las divisiones para el dictado de la clase respectiva.

Cronograma: Las actividades darán inicio en el mes de setiembre y constarán de una (1) clase semanal finalizando tentativamente en el mes de noviembre. 


\section{Temas}

1. Introducción: La cultura de la tributación. ¿Por qué un programa de Formación Tributaria? El Estado Nacional, Provincial y Municipal.

2. Breve noción de la estructura municipal. El Gobierno Municipal. Autonomía. Normas tributarias.

3. Constitución Nacional. Ley Orgánica de las Municipalidades.

4. Principios constitucionales provinciales y nacionales. ¿De dónde surge un tributo?

5. Código Tributario Municipal: concepto de tasa, servicio público, contribución por mejoras o reembolso.

6. El contribuyente. Los tributos. Concepto. Elementos. Clasificación. Tributos que cobra un Municipio.

7. La tasa municipal. La contribución por mejoras. Concepto. Diferencias. EI porqué de las tasas municipales.

8. Funciones del Estado. Necesidades. Recursos. Gasto público. Los servicios Públicos.

9. El presupuesto público. Importancia. ¿Para qué sirve? Responsabilidad tributaria. Valores.

El Programa de Formación Tributaria no solo pretende involucrar a los adolescentes en la realidad tributaria de su Municipio, sino al mismo tiempo, recompensarlos por su participación con un importante premio incentivo a quienes demuestren haber incorporado los conceptos tributarios vertidos en las clases impartidas, en oportunidad de una competencia entre escuelas y/o trabajo final a realizar tentativamente durante el mes de noviembre del año en curso, y que será informado promediando la ejecución del programa.

\section{Discusión}

Durante el desarrollo de la investigación se observó la importancia del rol que juega la educación en la formación del individuo, ejerciendo un alto nivel de influencia en la conducta de éste. La educación se tiene que comprender no solo como el acto material de transmitir conocimiento sobre diferentes áreas del saber, si no que va más allá, pues la aprehensión de estos conocimientos por el individuo van a contribuir el sistema educativo, pero este no actúa aisladamente, sino que lo hace en conjunto con el medio social del que forma parte el individuo.

Al respecto Pérez A. (1991), señala:

El educar se entiende como algo más sublime e importante que transmitir saberes o conocimientos, legitimaciones, ideologías, fórmulas científicas, explicaciones de procesos naturales. Educar es contribuir a formar hombres, pero la formación no solo proviene del sistema educativo, si no también está en manos del medio social, de la televisión, de los políticos, de los comerciantes, de ciertas personalidades, etc. (p. 394).

De lo expuesto, se infiere que el sistema educativo se va a concebir como una invitación al descubrimiento y al autodesarrollo, en definitiva hace referencia al ser íntimo de la persona, a la conducta, a los valores. Esta conducta, valores y creencias interactúan entre sí en el individuo dando origen a su cultura, ésta puede ser compartida por integrantes de un grupo humano, cuando estos poseen características semejantes.

La cultura es aprendida y compartida, el hombre no hereda la mayor parte de sus modos habituales de comportamiento, lo adquiere en el curso de su vida. Los hábitos adquiridos en la niñez siguen las pautas de 
niños, los padres, maestros y demás responsables de la educación y formación de los nuevos miembros de la sociedad les transmiten sus propias pautas de conducta, trasladando a cada nueva generación los conocimientos, habilidades, valores, creencias y actitudes, las definiciones antes citadas guardan relación entre sí, en cuanto manejan elementos comunes, tales como: conocimientos, comportamientos, sociedad, donde el actor principal es el hombre.

La Organización para la Educación, la Ciencia y la Cultura de las Naciones Unidas (UNESCO) 2006, define La cultura, como:

El conjunto de rasgos distintivos,
espirituales y materiales,
intelectuales y afectivos, que
caracterizan a una sociedad o grupo
social, engloba no solo las artes y las
letras, sino también los modos de
vida, los derechos fundamentales
del ser humano, los sistemas de
valores, las tradiciones y las
creencias. (p. 24)

La cultura es una manera de vivir juntos, moldea nuestro pensamiento, nuestra imagen y nuestro comportamiento. De igual modo es hacer, pensar, construir, edificar, derrumbar, obrar con la inteligencia, con la mano y con sus extensiones, las herramientas, la técnica que en su increíble crecimiento constituye ya una supranaturaleza.

En relación a la cultura tributaria, la cual representa una parte de la cultura nacional, va a tener un contenido individual al constituir en la creencia intima que tiene cada persona miembro de una agrupación social, que el impuesto constituye una aportación justa imprescindible y de provecho al ser utilizado para satisfacer las necesidades de la colectividad de la cual forma parte. A su vez, existe un hecho importante como es, de mayor transcendencia que la individual, al juzgar la sociedad la conducta del contribuyente evasor como un acontecimiento hacia la colectividad.

De lo anteriormente expuesto sobre educación, cultura tributaria, se puede inferir que la formación de la cultura tributaria debe iniciarse desde muy temprana edad en la persona, donde la educación juega un rol importante, para que éste adquiera la creencia firme de cumplir con sus obligaciones tributarias en el futuro, pero el aspecto más trascendental es lograr que esta cultura individual tenga una expresión colectiva, de manera que los individuos como grupo social no van a emitir un juicio interno de su propia conducta, sino que van a juzgar la de los individuos que integran al grupo, que comparte sus valores, creencias, principios y conducta en el cumplimiento de sus obligaciones en la sociedad.

La existencia de una cultura tributaria, cualquiera sea el grado que haya alcanzado, facilita el cumplimiento de los deberes formales, pero no puede pensarse que ella sea suficiente para asegurar la correcta percepción de los tributos. Aún en el caso de haber alcanzado un alto nivel, bastaría que unos pocos individuos se sustrajeran en el cumplimiento de su obligación para generar una desmoralización colectiva de los contribuyentes cumplidores y así destruir cualquier efectivo sistema de recaudación del tributo.

Por consiguiente, se debe mencionar que la existencia de una cultura tributaria, entre otros aspectos facilita el cumplimiento de los deberes formales de los contribuyentes y por ello es necesario y conveniente su formación y desarrollo en el ámbito nacional y organizacional, que constituye un factor de alto valor para lograr 
de manera voluntaria el aporte de los ciudadanos a los ingresos ordinarios por concepto de tributos.

Desde el punto de vista educativo, Viera

J. (1.997), señala:

La instrucción del venezolano en esta materia, es un medio para la formación de una cultura tributaria, debido a que el tributo como tal es de por sí, un concepto elaborado, complejo, que no resultará comprensible sino en la medida en que exista un nivel mínimo de instrucción, que brinde un grado elemental de receptividad. Esta tarea de formación debe iniciarse desde temprana edad y no es posible esperar resultados en el corto plazo, con lo cual, no se quiere decir que sea una tarea que debe posponerse, por el contrario, la decisión de hacerlo significará que deja de ser una actividad a plazo indefinida, para convertirse en una a largo plazo. (p. 44).

La citada autora, al igual señala que de existir esta cultura tributaria, le corresponde al Estado a través de sus organismos competentes el mantenerla, y de no existir es deber del Ministerio de Educación, orientar hacia su formación. Al igual ésta señala, que se debe realizar una formación en materia tributaria a todos los niveles de educación, logrando simultáneamente la formación educativa tributaria en los futuros contribuyentes y orientar e instruir a las actitudes, a través, de los medios de difusión disponible, esto representaría un mecanismo de acción efectivo que permitiría lograr el cumplimiento tributario actual.

La información antes señalada, es de gran importancia para la Unidad Educativa San Miguel Febres Cordero, ya que la educación es el medio a través del cual se genera un profundo cambio en el accionar de los ciudadanos, estimulando la participación corresponsable de los individuos para mejorar la calidad de vida del venezolano, de donde surge la necesidad de una relación entre el sector educativo y la administración tributaria, la cual debe desarrollarse en forma armónica, con respeto a los principios constitucionales y una actitud apegada a valores éticos entre los actores participantes. El aprendizaje de estos conceptos es necesario como forma de articulación interinstitucional y en la construcción de una nueva ciudadanía.

\section{La cultura tributaria}

Para hablar de cultura tributaria se tendría que hablar primero de impuestos, aunque se le dan varios nombres: tributos, contribuciones, arbitrios, derechos, ayuda, todos estos con diversas cualidades $y$ proporciones según las prácticas económicas y políticas imperantes en determinada época y en cada contexto, dado que el Impuesto: es un tributo absolutamente obligatorio, cuyo fundamento está basado en el beneficio general que el particular obligado recibe de la acción estatal tendiente a formar una infraestructura de orden social de relaciones interpersonales, de organización comunal en materia de educación, higiene, economía, obras y servicios públicos.

Entonces los impuestos se aplican al ciudadano, están regulados por las leyes, se pagan en dinero y constituyen formalmente el soporte material de la cosa pública. De manera que la receta desde el estado, luce así: Cultura Tributaria = Pagar Impuestos.

Ahora bien, existen autores que definen la cultura tributaria entre ellos, Golía (2003: 77), de la siguiente manera: "Es el conjunto de conocimientos, valoraciones y actitudes referidas a los tributos, así como al nivel de creencia respecto de los deberes y derechos que derivan para los sujetos activos y pasivos 
de esa relación. Ante lo relacionado en la cita del párrafo anterior, se puede conceptuar que la cultura es el conjunto de conocimientos, valoraciones y actitudes de un determinado grupo social, la cultura tributaria se refiere al conjunto de conocimientos, valoraciones y actitudes referidas a los tributos, así como al nivel de conciencia respecto de los deberes y derechos que derivan para los sujetos activos y pasivos de esa relación tributaria.

Corredor Corrales, M.Y y Díaz García (2007) "La Cultura Tributaria Municipal. Diagnóstico en la Parroquia El Valle del Municipio Bolivariano del Distrito Capital". Sostienen que la cultura debe verse como un conjunto de condiciones de creación y recepción de mensajes y de significaciones mediante los cuales el individuo se apropia de las formas que le permiten estructurar su lenguaje y su visión de mundo como instrumentos de interpretación para todos los temas relacionados con la agenda pública o la vida político-institucional. Por otra parte, la cultura tributaria es entendida como el conjunto de valores, creencias y actitudes compartidos con una sociedad respecto a la tributación y las leyes que las rigen, lo que conduce al cumplimiento permanente de los deberes fiscales.

La cultura tributaria aporta al presente trabajo una gran cantidad de información como valores, creencias que deben poseer cada persona y a la vez siendo útil para la alcaldía que conlleven a un mejor cumplimiento de los deberes tributarios y al compromiso de los ciudadanos. Con la cultura tributaria se pretende que los individuos de la sociedad involucrados en el proceso, tomen conciencia en cuanto al hecho de que es un deber constitucional aportar al Estado y comunicar a esa colectividad que las razones fundamentales de la tributación son el proporcionar a la
Nación los medios necesarios para que éste cumpla con su función primaria como lo es la de garantizar a la ciudadanía servicios públicos eficaces y eficientes

\section{Los tributos}

Villegas (1992) establece que:

Los tributos son entendidos
como "las prestaciones en
dinero, que el Estado exige en
ejercicio de su poder de
imperio, en virtud de una ley y
para cubrir los gastos que le
demandan el cumplimiento de
sus fines". De ahí, que son
considerados como una de las
fuentes de ingresos públicos
que poseen los Estados
adicional a otras actividades
financieras relacionadas
directamente con las ventajas
naturales, tecnológicas o
culturales que le son propias. (p.
23).

De acuerdo a lo que estipulan las citas up- supra se puede concluir que el tributo es una obligación que tiene toda persona natural o jurídica para colaborar con el gasto público y así el mismo pueda satisfacer las necesidades colectivas.

De lo anterior se tiene que todos los ciudadanos están obligados a ayudar al Estado a cubrir la carga pública, para ello se crean mecanismos legales como los tributos; los cuales deben estar establecidos en leyes que amparen las exigencias del Estado. Es de destacar que tales tributos irán surgiendo o modificándose de acuerdo a las condiciones económicas y sociales en las que se encuentre el país donde se desee entablar, obligando a todos cuantos estén incluidos dentro del régimen tributario de la nación, al pago de los mismos y al cumplimiento de las diferentes obligaciones que se impongan. 


\section{Educación tributaria}

Según el portal de internet en educación fiscal, Implementado por la oficina de euro social Fiscalidad, la cual tiene como objetivo fomentar el intercambio de experiencias entre instituciones y organizaciones de la Unión Europea y América Latina que trabajan en la consolidación de la educación fiscal como política pública, define la educación fiscal como:

Una política de Estado que tiene como objetivo fomentar una ciudadanía participativa y consciente de sus derechos y obligaciones. Se trata de un proceso de enseñanza y aprendizaje basado en tres ejes: formación en valores, construcción de la ciudadanía y cultura fiscal.

Por su parte, Delgado (2005) citado por la referida oficina de euro social Fiscalidad, establece que:

El sistema educativo puede preparar a los jóvenes para el momento en que deban cumplir sus obligaciones como contribuyentes, impartiendo una serie de conocimientos básicos que expliquen el sentido, el alcance y la finalidad de los impuestos y que describan brevemente los capítulos del Presupuesto público, así como los requerimientos más simples del sistema fiscal. Es importante que interioricen la idea de la fiscalidad como uno de los ámbitos donde se articula la necesaria correspondencia legal y ética entre derechos y obligaciones, donde se imbrican los intereses personales y los beneficios comunes. Para esto ni se puede ni se debe esperar a que los ciudadanos sean adultos. ( $p$. 394).
El Servicio Nacional Integrado de Administración Aduanera y Tributaria (Seniat), lleva a cabo un programa de educación tributaria, cuyo objetivo se basa en formar, promover y difundir estrategias didácticas para abordar la conciencia aduanera y tributaria a los docentes y estudiantes del Sistema Educativo Bolivariano, así como comunidades y habitantes en todo el territorio nacional. Con base en dicho programa, el SENIAT suscribió un convenio de cooperación interinstitucional con el Ministerio del Poder Popular para la Educación, a los fines de ejecutar acciones orientadas a reforzar la cultura aduanera y tributaria para favorecer la formación de valores ciudadanos.

Según la oficina de divulgación tributaria y aduanera (infonoticias 2007), el SENIAT consolida proceso de educación tributaria, por su parte el organismo tributario, a través de sus gerencias regionales de tributos internos $y$ de aduanas, ha venido desarrollando una serie de talleres con los docentes de los distintos distritos escolares de todo el país, con miras a la incorporación de la temática tributaria en los pensum de estudios de la educación básica.

Se puede decir que con la educación tributaria se pretende que los individuos de la sociedad involucrados en el proceso, tomen conciencia en cuanto al hecho de que es un deber constitucional aportar al Estado y comunicar a esa colectividad que las razones fundamentales de la tributación son el proporcionar a la Nación los medios necesarios para que éste cumpla con su función primaria como lo es la de garantizar a la ciudadanía servicios públicos eficaces y eficientes. 


\section{CONCLUSIONES}

En función al análisis de resultados y sobre la base de los objetivos de la investigación se establecen las siguientes conclusiones: En cuanto al objetivo uno el cual consistió en diagnosticar el nivel de conocimiento que poseen los niños $y$ adolescentes en materia tributaria de la Unidad Educativa San Miguel Febres Cordero, se concluyó que los jóvenes tienen poco conocimiento en materia tributaria, observándose desinterés por el tema tributario, esto es debido a que no existen programas de educación tributaria en ninguno de sus niveles de educación, ni se incentiva ni se fomenta en la comunidad la cultura tributaria para el pago de impuestos.

Sin embargo, señalan que el pago de impuestos ayuda a mejorar su bienestar social, ya que es una contribución para el desarrollo que se vincula con el bienestar de la colectividad, por lo tanto como futuros contribuyentes manifiestan que deben involucrarse en cuestiones tributarias, que se asuman como pagadores de impuestos y se conviertan, en consecuencia, en agentes efectivos del cambio social.

Por otra parte, en cuanto al objetivo dos, se describieron los aspectos que impulsan la cultura tributaria en la Unidad Educativa San Miguel Febres Cordero, concluyendo que no recibe orientación acerca de sus deberes formales, ya que se observa la falta de divulgación de las ordenanzas que rigen los impuestos. Asimismo, no tienen conocimiento si existen mecanismos de difusión aplicados por la Dirección de Hacienda para promover el conocimiento de los deberes tributarios, ya que no realizan campañas de divulgación de deberes tributarios. Por lo tanto, no se fomenta la cultura tributaria en el contribuyente para mejorar los niveles de recaudación Al respecto, están de acuerdo en recibir información en materia tributaria.

En este sentido, de acuerdo al objetivo tres, se concluyó que es necesario diseñar herramientas de Formación Tributaria para los estudiantes de la Unidad Educativa San Miguel Febres Cordero, ubicada en el Municipio Los Guayos, Estado Carabobo, por lo cual se elaboraron estrategias para la formación de valores ciudadanos a través de la educación tributaria, se impulsaron mecanismos de divulgación y promoción para los procesos tributarios y se estableció un programa de formación, orientación y cultura tributaria para los estudiantes de la Unidad Educativa San Miguel Febres Cordero.

\section{REFERENCIAS}

Armas M. y Colmenares M. (2009). Educación para la Cultura Tributaria. Revista Electrónica de Humanidades, Educación y Comunicación Social, Edición № 6, Año 4, de la Universidad Rafael Belloso Chacín

Belisario, H. (2002). Curso de finanzas, derecho financiero y tributario. Actualizada y Ampliada. Editorial Astrea. Buenos Aires, 285

Código Orgánico Tributario (2014). Gaceta Oficial de la República Bolivariana de Venezuela № 6.152 de fecha 18 de Noviembre de 2014

Código Orgánico Tributario. Gaceta Oficial N ${ }^{\circ}$ 37305 del 17 de Octubre de 2001

Constitución de la República Bolivariana de Venezuela (1999). Gaceta Oficial de la República Bolivariana de Venezuela № 36.860 Extraordinaria de 30 de diciembre de 1999, reimpresa en Gaceta Oficial № 5.453 de fecha 24 de marzo de 2000

Corrales, M., \& García, W. (2007). La cultura tributaria municipal. Diagnóstico en La parroquia El Valle del municipio Bolivariano del Distrito Capital. Observatorio de la Economía Latinoamericana, 81 
Delgado Y. (2006). La Investigación Social en Proceso: Ejercicios y Respuestas. Segunda Edición. Blue Cube Digital Services. Valencia Venezuela

García Hoz, V y Medina, R. (1988). Organización y Gobierno de Centros Educativos. Editorial quinto centenario. Segunda edición. Bogotá

Golía 2.003. Como Controlar la Evasión. Revista Dinero

Guaiquirima C. (2004). Cultura Aduanera y Tributaria. Temas Tributarios, folleto № 2, Oficina de divulgación Aduanera y Tributaria (SENIAT). (P. 12)

Hortta Vásquez E. y Rodríguez G. (1994). Ética General. Ecoe Ediciones, Universidad Católica de ColombiaBogotá

Méndez M. (2004). Cultura Tributaria, deberes y derechos Vs Constitución de 1999
Méndez, C. (2010). Metodología, Diseño y Desarrollo del Proceso de Investigación. Editorial Mc.Graw Hill. Colombia

Moya E (2009). Elementos de Finanzas Públicas y Derecho Tributario. Editorial Mobi - Libios. Caracas 2da edición

Palella, S. y Martins, F. (2012). Metodología de la Investigación Cuantitativa. Editorial Fedupel. Venezuela. Revista Espacio Abierto. Vol. 13, № 1, eneromarzo (p. 123-137)

Viera, J. (1997). Relación entre la asistencia tributaria suministrada por la contribuyente de la gerencia regional de tributos internos región Centro Occidental - Barquisimeto y el conocimiento de las obligaciones tributarias por parte de los contribuyentes. Trabajo de grado Universidad Centro Occidental Lisandro Alvarado (UCLA). Barquisimeto 